\title{
BMJ Open Quality Quantifying disruption of workflow by phone calls to the neuroradiology reading room
}

\author{
Shyam Sabat, Paul Kalapos, Einat Slonimsky
}

To cite: Sabat S, Kalapos $P$, Slonimsky E. Quantifying disruption of workflow by phone calls to the neuroradiology reading room. BMJ Open Quality 2019;8:e000442. doi:10.1136/ bmjoq-2018-000442

Received 1 June 2018 Revised 12 August 2019 Accepted 18 August 2019
Check for updates

(c) Author(s) (or their employer(s)) 2019. Re-use permitted under CC BY-NC. No commercial re-use. See rights and permissions. Published by BMJ.

Penn State Health Milton S Hershey Medical Center, Hershey, Pennsylvania, USA

Correspondence to Dr Einat Slonimsky; einatslow@gmail.com

\section{ABSTRACT}

Introduction The purpose of this study was to understand the source and the reason for the phone calls to our neuroradiology suit and to quantify the size of the problem in terms of duration of individual and aggregated calls.

Materials and methods Observation of the neuroradiology reading room for the entire duration of the working hours over three non-consecutive days was performed, and included telephone calls start time, end time and calls duration for incoming telephone calls. After each phone call the recipients were queried on the details of the phone call; the origin of the call, the reason for the call and the response.

Results The average total number of minutes (min) spent on the phone each day was 64 min per working day with a total of 39 phone calls per day and 4.4 per hour on average. The trainees answered $71 \%$ of the phone calls with additional intervention by attending in $13 \%$ of phone calls. The most common source of phone calls was from either the MRI/CT technicians (48\%), followed by providers (20\%) and returning pages (18\%).

Conclusion Cumulative time spent on the phone by neuroradiologists in the reading room ended up in more than an hour per working day, while trainees were taking the majority of phone calls. Most phone calls originated from technicians, hence, requiring specific solutions to mitigate this kind of interruption.

\section{INTRODUCTION}

Clinical work is many times interrupted for justified and unjustified reasons, ${ }^{1}$ and it is known as a major source of frustration among clinicians and a threat to patient safety. ${ }^{23}$ The effects of interruptions have been linked to errors in many clinical settings including nursing, ${ }^{4-6}$ the operating room ${ }^{7}$ and in the radiology reading rooms of diagnostic radiologists. ${ }^{8}$ Recent publications were able to validate the negative effect of interruptions to the radiology reading rooms on the diagnostic accuracy of radiologists, ${ }^{9-12}$ especially on the on-call radiologist. ${ }^{13-15}$

Nowadays, diagnostic radiologists are required to simultaneously perform several tasks while interpreting a study, including vetting requests, protocoling studies, consenting patients, making contrast calculations, administrating contrast and in person clinical discussions with other physicians. Many of these responsibilities are fulfilled by phone calls to the neuroradiology suit and cause an interruption of the radiologists' workflow.

With phone calls being one of the most frequent interruptions to the workflow of radiologists, previous studies have quantified the disruptive nature of these phone calls in terms of the effect on performance of the radiologists. ${ }^{189141617}$ However, none of these studies were looking to evaluate the nature of the disturbances themselves and the source. Being able to evaluate the source of the frequent phone calls to the neuroradiology reading room, would possible allow future interventions and reduction in the load of interruptions. Therefore, the purpose of this study was to quantitate the load and origin of phone call distractions to our neuroradiology suit.

\section{MATERIALS AND METHODS}

The institutional review board waived the requirement for informed consent. Patients were not involved in the current study.

\section{Patient and public involvement}

Patients were not involved in the current study.

\section{Data collection}

During the study period, a fourth-year medical student observed the neuroradiology reading room during weekdays for the entire duration of the working hours (08:00-17:00 hours), including the lunch interval. The neuroradiology reading room in our tertiary hospital included seven working stations and was occupied by at least an attending radiologist and a trainee (resident or neuroradiology fellow). Data were collected over three non-consecutive days in order to remove the bias of a single day or a particular day of the week. 
Phone call start time, end time and call duration of incoming telephone calls were collected. After each phone call, the recipients were inquired on the details of the phone call; the origin of the call, the reason for the call and the response. Time duration between phone calls and an overall average were calculated daily.

The source of the call included either an MRI technician, a CT technician, an X-ray technician, a phone call from a provider, a return page phone call or others.

Our neuroradiology reading room includes on average two attendings, two resident and a neuroradiology fellow. The residents and the neuroradiology fellow answer the phone calls during the working hours (08:00-17:00) on weekdays except for lunch break (12:00-13:00 hours), which is covered by an attending. Phone calls which were eventually referred to an attending by the resident or a fellow were considered as one phone call.

The reciepient was noted as either a trainee (resident/ fellow) or an attending radiologist (all attending in our institution are fellowship trained neuroradiologists with at least 5 years' experience). All incoming phone calls, including physicians who were returning pages, were included.

Reasons for incoming calls were classified as asking for a request to be vetted (urgent study), asking for a study to be reported, questions regarding study protocol, asking for a second review of a study, discussion of the study of choice, return of a page and others.

Calls made were excluded from statistical analysis. The data were recorded and analysed using Excel. We considered a $\mathrm{p}$ value $<0.05$ as statistically significant.

\section{RESULTS}

The average total number of minutes spent on the phone each day was $64 \mathrm{~min}$ per working day (9hours). The average total phone calls per day were 39 with 4.4 phone calls per hour on average and an average duration of each call of $1 \mathrm{~min} 39 \mathrm{~s}$ (figure 1). The average total duration of phone calls not including return pages was $48 \mathrm{~min}$ per work day, with an average of $1 \mathrm{~min}$ and $35 \mathrm{~s}$ per phone call.

The trainees (residents/fellows) answered $71 \%$ of the phone calls while an attending answered $29 \%$ of the phone calls. Additional intervention by an attending was required in $13 \%$ of phone calls answered by residents.

The most common source of phone calls to the neuroradiology reading room was from either the MRI/CT technicians (48\%), when phone calls from MRI technicians were more prevalent $(28 \%)$. Phone calls from providers were also common $(20 \%)$ while phone calls from X-ray technicians $(9 \%)$ and others (5\%) were less common. Returning pages resulted in phone calls coming from the neuroradiology reading room in $18 \%$ of the phone calls.

The average duration of received calls by residents was 1 min $26 \mathrm{~s}$ and that by an attending was 2 min $2 \mathrm{~s}$. The average interval between phone calls from all sources was $13 \mathrm{~min}$ and $37 \mathrm{~s}$. The shortest average interval between calls originating from a single source was from MRI/CT technologists and was $27 \mathrm{~min}$ and $17 \mathrm{~s}$ while the second shortest average interval were calls originating from providers and was $38 \mathrm{~min}$ and $18 \mathrm{~s}$. The average duration between phone calls was 13:31 min (13:52, 13:00, 13:43 for days 1, 2 and 3, respectively).

Fluctuation of phone calls during the day was similar in days 1 and 3 with phone calls being more prevalent at the end of the day while at day 2 phone calls were more prevalent in midday (Figure 1).

The most prominent reason for phone calls included discussion about the study protocol $(27 \%)$, request for an urgent review of a study (22\%) and returning a page

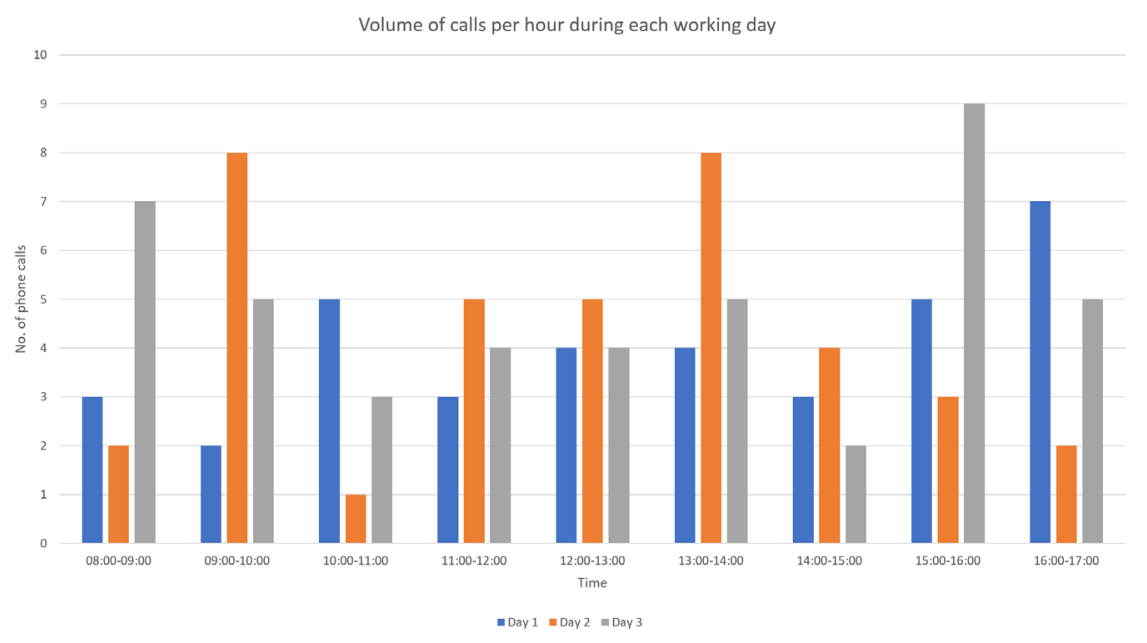

Figure 1 Volume of calls per hour for each of the working days being evaluated. 
Table 1 Major causes of interruptions and possible solutions

\section{Communication with technicians}

\section{Protocol management Instant messaging integrated with PACS (eg, Primordial Software).}

Designated radiologist to handle exam-related issues with a dedicated room to reduce the load of calls to the reading room.

Empowering technologists to have decision-making capability.

Communication with physicians

Request for urgent review of study Electronic alert system to request for an urgent review.

Clinical consultation

Secure encrypted messaging apps for communication (like WhatsApp, TigerConnect).

Rotating radiologist on-call for all clinical consultations.

(18\%). Less frequent phone calls were made regarding a second review of a study (12\%), request for an urgent study $(4 \%)$, discussion with a physician of the optimal study required $(2 \%)$ and others $(15 \%)$.

\section{DISCUSSION}

Phone calls interruptions to clinical workflow have been recognised as a problematic distraction in radiology reading rooms ${ }^{3}$ due to time consumption as well as disrupting the 'train of thought' of the involved radiologist and others in the same room. ${ }^{18}$

Our results demonstrated that the highest load of interruptions to the neuroradiology suit resulted from two main sources: technicians and referring physicians; this was demonstrated both when analysing the origin of the phone calls and the reason for the phone calls.

Identifying the source of the multiple interruptions is crucial to form potential solutions and modifications of the workflow in order to reduce the load of interruptions. The process of changing the load of interruptions include combining modifications to reduce the interruptions and modifications to reduce the nature of the interruptions and make them less interruptive (table 1).

Communication with the technicians is extremely important in order to maintain high quality of studies. Many times, as was reflected in our study, the technicians would consult the radiologist regarding protocoling a study or slight modification of the imaging protocols in order to optimise the imaging study. Electronic messaging integrated with PACS (eg, Primordial Software) is available between the technicians and the radiologists, allowing free communication and reducing the number of unusual cases that the technician would require to call the radiologist with a specific question. But these 'unusual cases' are often very frequent suggesting that the full benefit of electronic-based communication is not used, and both technicians and radiologists rely on phone call communication. Given that the majority of the calls originated from MRI/CT technologist and calls from this origin had the shortest interval, potential solutions include the use of an instant messaging chat. Advocating this approach among technologists would allow to increase this type of communication rather than having to call in the reading room. One can argue that this interruption would only reduce the load of phone call and not the load of individual interruption. However, usually, protocoling a study is not urgent and can be done between cases, which would not interfere with 'train of thought' and make the interruption less interruptive.

Having a designated radiologist to handle exam-related issues, preferentially in a segregated reading room, is another solution to reduce the load of the phone calls to the reading room and to reduce the individual load of interruptions.

Empowering CT and MRI technologists to have more decision-making capability within the limits of their training is paramount to reduce the load of phone calls to the reading room. Another method to mitigate phone calls from technicians can be direct instructions given by the radiologist in the patient's file, or a referral for a specific predetermined protocol. We did not look into the seniority of the technicians who made the phone calls, but it is possible that majority of phone calls were made from junior technicians who needed more assistance in decision making. Further research may look at the impact of any of these interventions on the reduction of incoming calls.

The communication with the referring physician is one of the most important of radiologist responsibility and is crucial for optimal patient care. Similar to previous studies, this type of communication resulted in phone calls load to the neuroradiology suit. Reason of call analysis revealed that the referring physicians were mostly inquiring about prioritising interpretation of studies. Hence, we suggest applying an electronic instant alert system that would allow the clinician to request for an urgent review of a study or a secure encrypted messaging application (like WhatsApp, TigerConnect).

A previous study has evaluated the appropriateness of these phone calls and found that half of the phone calls were not appropriate according to the radiologists. ${ }^{1}$ Since this type of specific communication is highly important, and yet, causing constant disruption, not without consequences, ${ }^{19}$ this requires the pursuit for a possible solution.

Our results indicate that the majority of phone calls were answered by residents who were more affected by 
those interruptions. A paper previously published in Academic Radiology in 2014 by Balint et al demonstrated that distractions impacted on on-call radiology residents' diagnostic accuracy with positive correlation between the amount of interruptions and the frequency of a major discrepancy. ${ }^{9}$ Another study has shown that radiology residents are especially vulnerable experiencing frequent disruptions while on call with an overall rate of major discordance approximately $1 \%-2 \% .^{8}$ The amount of phone call interruptions shown in the study by Balint $e t$ al are similar with the amount of phone calls in our study.

Interruptions often resulted from clinical consultation by the treating physicians. Since the communication between the physician and the radiologist is imperative for patient care, we believe this kind of interruption should not be eliminated. We suggest a potential solution of a rotating attending physician on-call who would be responsible for all clinical consultations. The same on-call attending can be in charge of providing urgent review of studies, hence, preventing other radiologists from being interrupted.

While no study has specified the time to read a film with and without an interruption, a study by Williams et al fount that interruptions cost an increase in task completion time. ${ }^{15}$ This only strengthens the need to find a solution to minimise the phone calls interruption in order to prevent prolonged interpretations time.

This study is a descriptive observational study with few limitations. This is a one-centre study with only three days observational reports. Moreover, we did not correlate the phone calls with reports mismatches.

\section{CONCLUSION}

Phone calls are a source of substantial disruption in the radiology reading room resulting in loss of more than an hour of a physician's time in a 9-hour work day. The majority of phone calls emerged from technicians and regarded protocoling an exam, hence, future solution should be focused in this direction. Trainees were mostly affected as they were responsible for answering most phone calls. Methods to decrease phone call distractions should be encouraged in radiology departments so as to increase turnaround time, improve residents' training and improve patients' safety.

Contributors SS: planning, data collection, manuscript editing. ES: data collection, statistical analysis, manuscript writing, submitting the manuscript. PK: manuscript writing.

Funding The authors have not declared a specific grant for this research from any funding agency in the public, commercial or not-for-profit sectors.

Competing interests None declared.

Patient consent for publication Not required.
Ethics approval This study protocol was reviewed and approved by the institutional review board.

Provenance and peer review Not commissioned; externally peer reviewed.

Data availability statement Data are available on reasonable request. All data relevant to the study are included in the article or uploaded as supplementary information.

Open access This is an open access article distributed in accordance with the Creative Commons Attribution Non Commercial (CC BY-NC 4.0) license, which permits others to distribute, remix, adapt, build upon this work non-commercially, and license their derivative works on different terms, provided the original work is properly cited, appropriate credit is given, any changes made indicated, and the use is non-commercial. See: http://creativecommons.org/licenses/by-nc/4.0/.

\section{REFERENCES}

1. Watura C, Blunt D, Amiras D. Ring ring ring! characterising telephone interruptions during radiology reporting and how to reduce these. Curr Probl Diagn Radiol 2019;48:207-209.

2. Froehle CM, White DL. Interruption and forgetting in KnowledgeIntensive service environments. Prod Oper Manag 2014;23:704-22.

3. Li SYW, Magrabi F, Coiera E. A systematic review of the psychological literature on interruption and its patient safety implications. J Am Med Inform Assoc 2012;19:6-12.

4. Stelfox HT, Palmisani S, Scurlock C, Orav EJ, et al. The "To Err is Human" report and the patient safety literature. Qual Saf Health Care 2006;15:174-8.

5. Blendon RJ, DesRoches CM, Brodie M, et al. Views of practicing physicians and the public on medical errors. $N$ Engl $J$ Med 2002;347:1933-40.

6. McGillis Hall L, Pedersen C, Fairley L. Losing the moment: understanding interruptions to nurses' work. J Nurs Adm 2010;40:169-76.

7. Arora S, Hull L, Sevdalis N, et al. Factors compromising safety in surgery: stressful events in the operating room. Am J Surg 2010;199:60-5.

8. JP Y, Kansagra AP, Mongan J. The radiologist's workflow environment: evaluation of disruptors and potential implications. Journal of the American College of Radiology : JACR 2014;11:589-93.

9. Balint BJ, Steenburg SD, Lin H, et al. Do Telephone Call Interruptions Have an Impact on Radiology Resident Diagnostic Accuracy? Acad Radiol 2014;21:1623-8.

10. Cooper VF, Goodhartz LA, Nemcek AA, et al. Radiology resident interpretations of on-call imaging studies: the incidence of major discrepancies. Acad Radiol 2008;15:1198-204.

11. Flink CC, Mueller JS, Kiproff PM. Impact of resident call eligibility on major discrepancy rate. Acad Radiol 2010;17:1299-301.

12. Miyakoshi A, Nguyen QT, Cohen WA, et al. Accuracy of preliminary interpretation of neurologic CT examinations by on-call radiology residents and assessment of patient outcomes at a level I trauma center. J Am Coll Radiol 2009;6:864-70.

13. Glover M, Almeida RR, Schaefer PW, et al. Quantifying the impact of Noninterpretive tasks on radiology report Turn-Around times. J Am Coll Radiol 2017;14:1498-503.

14. Ratwani RM, Wang E, Fong A, et al. A human factors approach to understanding the types and sources of interruptions in radiology reading rooms. Journal of the American College of Radiology 2016;13:1102-5.

15. Williams LH, Drew T. Distraction in diagnostic radiology: how is search through volumetric medical images affected by interruptions? Cogn. Research 2017;2.

16. Kansagra AP, Liu K, Yu J-PJ. Disruption of radiologist workflow. Curr Probl Diagn Radiol 2016;45:101-6.

17. Levin DC, Rao VM, Parker L, et al. Analysis of radiologists' imaging workload trends by place of service. J Am Coll Radiol 2013;10:760-3.

18. Wilcox L, Patel R, Chen Y, et al. Human factors in computing systems: focus on patient-centered health communication at the ACM SIGCHI conference. Patient Educ Couns 2013;93:532-4.

19. Altmann EM, Gray WD. An integrated model of cognitive control in task switching. Psychol Rev 2008;115:602-39. 